\title{
Mutation of Inositol 1,3,4-trisphosphate 5/6-kinase6 Impairs Plant Growth and Phytic Acid Synthesis in Rice
}

\author{
Meng Jiang ${ }^{1,2,3} \mathbb{D}$, Yang Liu ${ }^{1}$, Yanhua Liu ${ }^{1}$, Yuanyuan Tan ${ }^{1}$, Jianzhong Huang ${ }^{1,3}$ and \\ Qingyao Shu $1,2, * \mathbb{D}$ \\ 1 National Key Laboratory of Rice Biology, Institute of Crop Sciences, Zhejiang University, Hangzhou 310058, \\ China; mengjiang@zju.edu.cn (M.J.); 21616041@zju.edu.cn (Y.L.); yanhual624@163.com (Y.L.); \\ tanyy@zju.edu.cn (Y.T.); jzhuang@zju.edu.cn (J.H.) \\ 2 Hubei Collaborative Innovation Center for Grain Industry, Yangtze University, Jingzhou 434025, China \\ 3 Institute of Nuclear Agricultural Sciences, Zhejiang University, Hangzhou 310058, China \\ * Correspondence: qyshu@zju.edu.cn; Tel.: +86-571-88982859
}

Received: 20 March 2019; Accepted: 24 April 2019; Published: 29 April 2019

\begin{abstract}
Inositol 1,3,4-trisphosphate 5/6-kinase (ITPK) is encoded by six genes in rice (OsITPK1-6). A previous study had shown that nucleotide substitutions of OsITPK6 could significantly lower the phytic acid content in rice grains. In the present study, the possibility of establishing a genome editing-based method for breeding low-phytic acid cultivars in rice was explored, in conjunction with the functional determination of OsITPK6. Four OsITPK6 mutant lines were generated by targeted mutagenesis of the gene's first exon using the CRISPR/Cas9 method, one (ositpk6_1) with a 6-bp in-frame deletion, and other three with frameshift mutations (ositpk6_2,_3, and _4). The frameshift mutations severely impaired plant growth and reproduction, while the effect of ositpk6_1 was relatively limited. The mutant lines ositpk6_1 and_2 had significantly lower levels $(-10.1 \%$ and $-32.1 \%)$ of phytic acid and higher levels (4.12- and 5.18-fold) of inorganic phosphorus compared with the wild-type (WT) line. The line ositpk6_1 also showed less tolerance to osmotic stresses. Our research demonstrates that mutations of OSITPK6, while effectively reducing phytic acid biosynthesis in rice grain, could significantly impair plant growth and reproduction.
\end{abstract}

Keywords: genome editing; growth; ositpk6; phytic acid; rice

\section{Introduction}

Myo-inositol-1,2,3,4,5,6-hexakisphosphate ( $\left.\mathrm{IP}_{6}\right)$, also known as phytic acid (PA), is the main storage form of phosphorous (P) (65-80\%) in cereal and legume seeds, accounting for $\sim 1.5 \%$ of the dry weigh [1]. In most cereal grains, PA exists as mixed salts (phytates) in protein storage bodies and can chelate several mineral cations, including $\mathrm{Zn}^{2+}, \mathrm{Fe}^{2+}, \mathrm{Ca}^{2+}$, and $\mathrm{Mg}^{2+}$ [2]. During seed germination, endogenous grain phytase is activated to degrade phytate, releasing myo-inositol, phosphorus, and bound mineral cations [3], which are utilized by the developing seedlings. The PA biosynthetic pathway is still not well defined, but a number of genes involved in its biosynthesis or transport have already been cloned in several plants. Mutations of these genes could result in low-phytic-acid (lpa) grains in rice [4-14] and other plants, e.g., wheat [15] and maize [3,16,17]. In rice, 12 genes have been identified that catalyze the production of intermediate inositol polyphosphates in seeds [18].

Inositol 1,3,4-trisphosphate 5/6-kinase (ITPK) plays a pivotal role in phytic acid biosynthesis, whereby the inositol triphosphate $\left(\mathrm{IP}_{3}\right)$ molecule is further phosphorylated at the 5 th or 6 th position $[19,20]$. ITPK belongs to the ATP-grasp fold proteins group [21] and is conserved from plants to humans with diverse functions. ITPK has even been found in the anaerobic protozoan Entamoeba histolytica [22], where 
its transcription is slightly induced by heat shock, demonstrating its role in the cellular response to stress [21]. The first plant ITPK, AtITPK1, was identified in Arabidopsis [20]. AtITPK1 is involved in photomorphogenesis possibly by interacting with the constitutive photomorphogenic (COP) signalosome under red light [23]. The kinase activity of AtITPK1 is indispensable for maintaining inorganic phosphorus (Pi) homeostasis under Pi-replete conditions, and itpk1 mutants exhibited decreased levels of $\mathrm{IP}_{6}$ and diphosphoinositolpentakisphosphate $\left(\mathrm{IP}_{7}\right)$. Disruption of another ITPK family enzyme, ITPK4, also caused depletion of $\mathrm{IP}_{6}$ and $\mathrm{IP}_{7}$ but did not display similar Pi-related phenotypes as itpk1 [24]. AtITPK4 is an outlier to its family and does not display inositol 3,4,5,6 tetrakisphosphate 1-kinase activity; rather, it displays inositol 1,4,5,6-tetrakisphosphate and inositol 1,3,4,5-tetrakisphosphate isomerase activity [21]. AtITPK2 was required for seed coat development and lipid polyester barrier formation [25], and ABA or phosphorus deficiency could induce AtITPK2 expression. In maize (Zea mays L.), ZmITPK1 exhibits multiple inositol phosphate kinase activities and is involved in phytic acid biosynthesis in developing seeds [17]. In soybean (Glycine max L.), GmITPK1 is a potential candidate for developing low-phytate soybean [26], and GmITPK2 may play a role as a dehydration and salinity stress regulator [27].

In rice (Oryza sativa L.), the OsITPK genes can be divided into three sub-families [18]. OsITPK1, OsITPK2, and OsITPK3 belong to subgroup I, each with 10 exons and 9 introns; OsITPK4 and OsITPK5 belong to subgroup II, which has no intron; and OsITPK6 belongs to subgroup III, with 12 exons and 11 introns. OsITPK2 is a negative regulator of osmotic stress signaling [28], and its disruption could affect the expression of some of its homologous genes, OsITPK1 and OsITPK4 [29]. The expression of OsITPK4, but not of OsITPK1, 2, 3, and 5 can be strongly induced by cold and heat stresses [29]. The $\mathrm{IP}_{3}$ level was not affected by the ositpk2 mutation, probably owing to redundant functions of other homologs [29].The expression of OsITPK6 could also be induced by heat [29], and mutations of OsITPK6 were already demonstrated to result in significant reduction of $\mathrm{IP}_{6}$ in rice grains [30], i.e., mutant lines with the amino acid substitution P522L had $\mathrm{IP}_{6}$ content about half that of the wild-type (WT) line. Among the ositpk6 mutants, one line with a P522L amino acid substitution had agronomic performance (seed weight, germination, and seedling growth) similar to that of its WT parent, suggesting OsITPK6 could be a desirable target of mutagenesis for breeding yield-competitive lpa rice [30]. Since the binding site for nucleotide or ATP is between 200 and 500 amino acids in OsITPK6, the substitution mutation (P522L) is localized outside of this binding region. The effect of the P522L mutation in OsITPK6 on $\mathrm{IP}_{6}$ biosynthesis could be related to the interaction of the enzyme with another substrate inositol polyphosphate. On the other hand, a splicing mutant of OsITPK6 at the 9th intron showed a more severe lpa phenotype: lower phytic acid content with reduced seed set [30]. Hence, it would be worthwhile to examine the function of OsITPK6 by generating more and different mutants, particularly by disruption of the ATP-binding region.

The clustered regularly interspaced short palindromic repeats (CRISPR) and CRISPR-associated protein 9 (Cas9) system, CRISPR/Cas9, is an efficient and precise genome-editing technique and has the potential to be used for crop improvement [31-33], including rice [34-36]. In the present study, we explored the possibility of establishing a genome-editing-based method for the fast breeding of yield-competitive lpa rice by evaluating OSITPK6 mutants generated by CRISPR/Cas9-mediated mutagenesis. Our results showed that mutation of OSITPK6 not only significantly reduced the accumulation of $\mathrm{IP}_{6}$ in rice grains but also impaired plant growth and tolerance to abiotic stress.

\section{Results}

\subsection{Mutations of OsITPK6 and Development of Homozygous Transgene-Free Mutant Lines}

In total, we obtained 23 hygromycin phosphotransferase (HPT)-positive $\mathrm{T}_{0}$ plants transformed with the CRISPR/Cas9 vector $\mathrm{pH}$-itpk6. Among them, seven plants were found mutated at the target region, which represents an editing efficiency of $30.4 \%$. $\mathrm{T}_{1}$ plants were tested for the presence of mutations and T-DNA, and transgene-free $\mathrm{T}_{1}$ plants with four types of mutation were identified. The mutations included a single-nucleotide (nt) insertion and three types of deletion (Figure 1A). Seeds 
were harvested from $T_{1}$ plants with different mutations and developed into homozygous mutant lines, which were designated ositpk6_1,_2, _3, and _4.

(A)

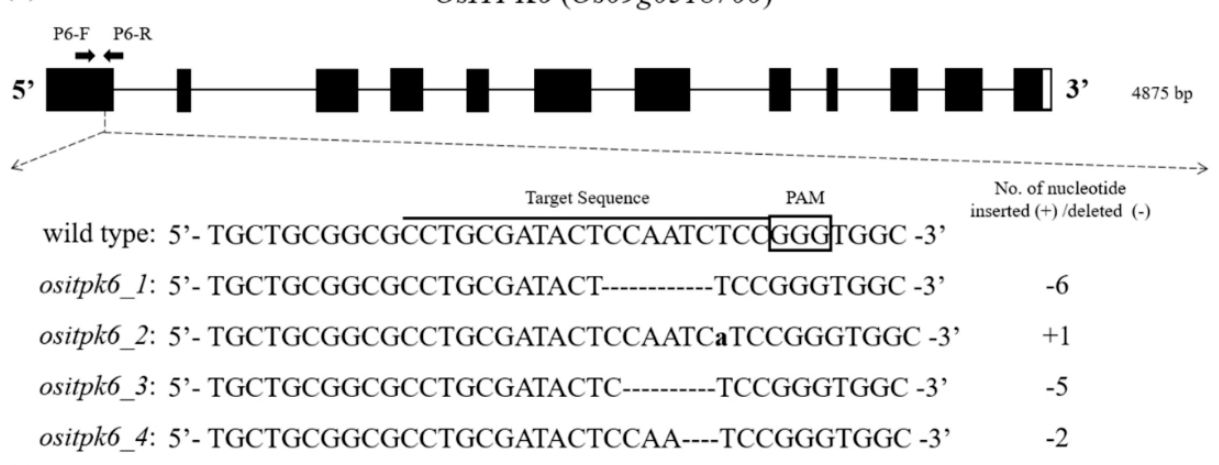

(B)

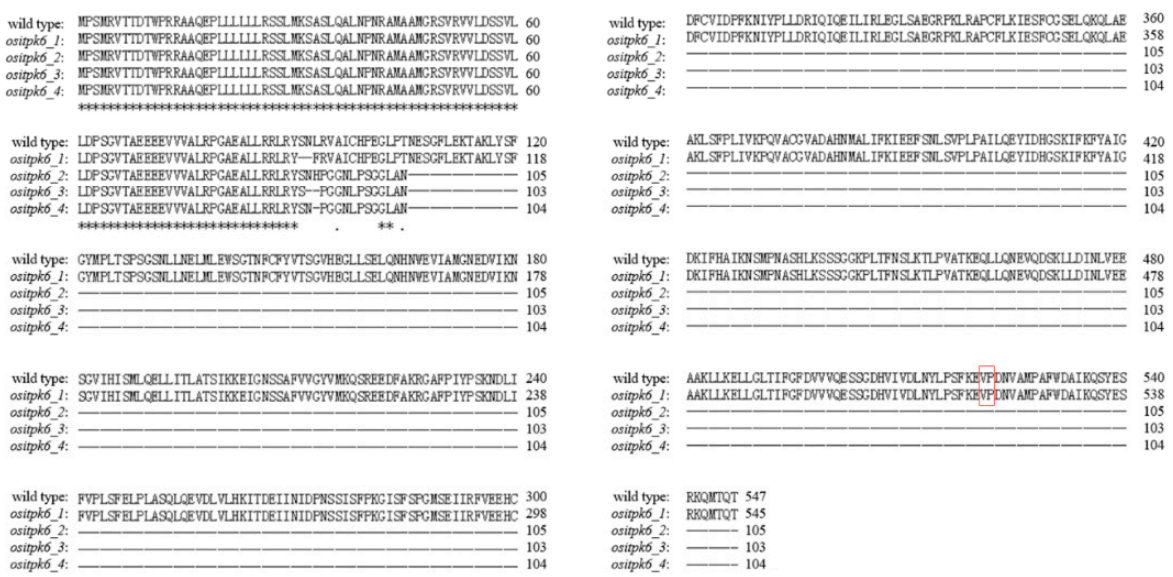

(C)

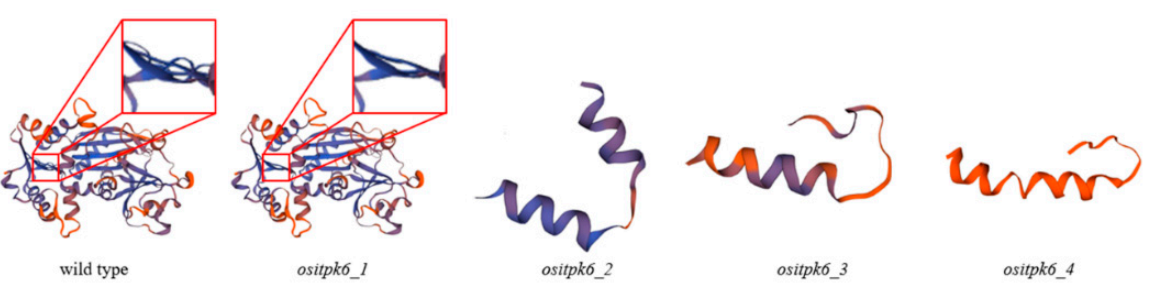

Figure 1. Schematic diagram of OsITPK6 and sgRNA target site for CRISPR/Cas9-mediated mutagenesis of OsITPK6, and prediction of the related wild-type and mutant proteins. (A) Exons, introns, and UTRs are indicated by solid boxes, lines, and blank boxes, respectively. P6-F and P6-R are primers for mutation genotyping, and their positions are indicated by arrowheads. Mutation identified within the target site of OSITPK6 generated through CRISPR/Cas9-mediated genome editing in rice. The PAM sequences (NGG) are boxed, and the 20-nt target sequences are underlined. Mutations are shown in lowercase letters (for insertions) or '-' (for deletions). (B) The amino acid sequences of mutant proteins were aligned to that of the wild-type protein using the Clustal Omega Multiple Sequence Alignment (https://www.ebi.ac.uk/Tools/msa/clustalo/). The numbers represent the total number of amino acids, and the amino acid V521 and P522 are highlighted in red box. (C) The three-dimensional structures of OsITPK6 and its mutants were analyzed on SWISS-MODEL (https://www.swissmodel.expasy.org/).

The ositpk6_1 (a 6-nt in-frame deletion) mutation would result in the loss of two amino acids at positions 91 to 92 (Figure 1B). In contrast, all the other three mutations, i.e., ositpk6_2 (a 1-nt insertion), ositpk6_3 (a 5-nt deletion), and ositpk6_4 (a 2-nt deletion), would generate a premature stop codon almost right after the mutation site and, hence, significantly shorten the encoded proteins (Figure 1B). Consequently, the ositpk6_2,_3 and _4 mutant alleles were predicted to produce proteins of only 105, 103, and 104 amino acids, respectively (Figure 1C). Analysis of ITPK6 proteins of six organisms indicated 
that the two amino acids missing in the ositpk6_1 mutant were located in a highly conserved segment (Figure S1), suggesting the mutation of ositpk6_1 could have a potential functional consequence.

\subsection{Impact of ositpk6 Mutations on Plant Growth and Seed Germination}

Plant growth of ositpk6_2,_3, and _4 was significantly impaired. First, their panicles were significantly shorter $(-30.1 \%,-28.8 \%$, and $-29.1 \%$, respectively) than that of the WT parental cultivar Xidao 1 (Figure 2A). Second, the mutant panicles had a high percentage of empty grains with darkened glumes (Figure 2B). Third, the height of the mutant plants was significantly reduced $(-37.5 \%,-36.9 \%$, and $-39.1 \%$, respectively) compared with that of their parental cultivar Xidao 1 (Figure 2C). The impact of ositpk6_1 on plant growth and seed set was limited and not obvious (Figure 2A-C). No significant differences of tiller number per plant were observed between Xidao 1 and all four ositpk6 mutant lines (Figure 2D). Compared with Xidao 1, the seed set and 1000-grain weight of ositpk6_1 were also significantly decreased $(-11.7 \%$ and $-10.8 \%$, respectively) (Figure $2 \mathrm{E}, \mathrm{F})$. Due to the extremely low seed set, we were not able to harvest enough seeds from ositpk6_3 and ositpk6_4 for the evaluation of 1000-grain weight and other characteristics. The germination of ositpk6_1 was slower in the first three days (Figure 3A) but gradually caught up with that of the WT after five days and reached $\sim 80 \%$ on the 7th day (Figure 3B). The germination rate of ositpk6_2 was far lower than that of the WT (Figure 3A), being only $\sim 20 \%$ on the 7 th day (Figure 3B).

(A)

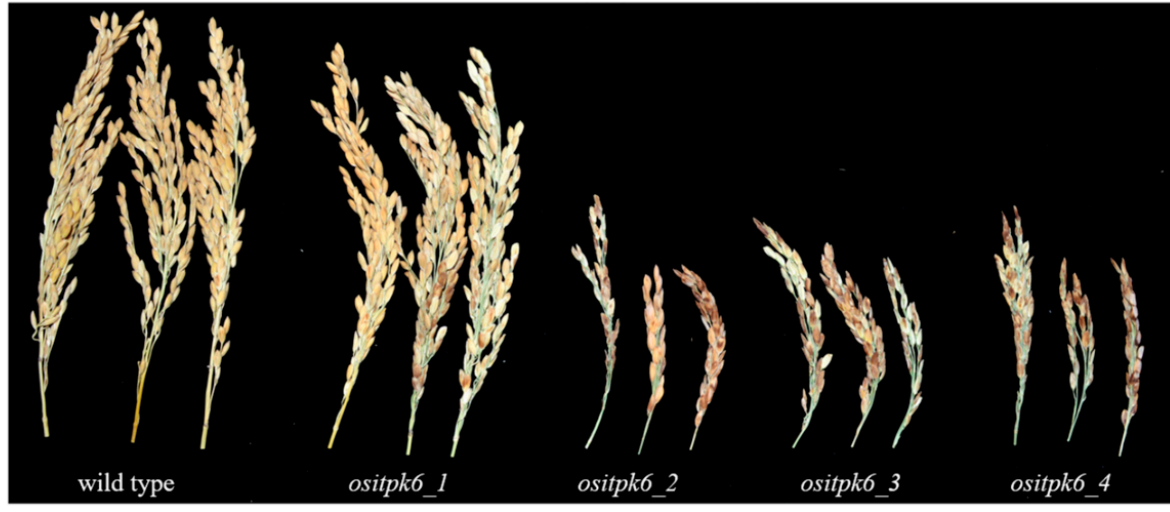

(B)

(C)
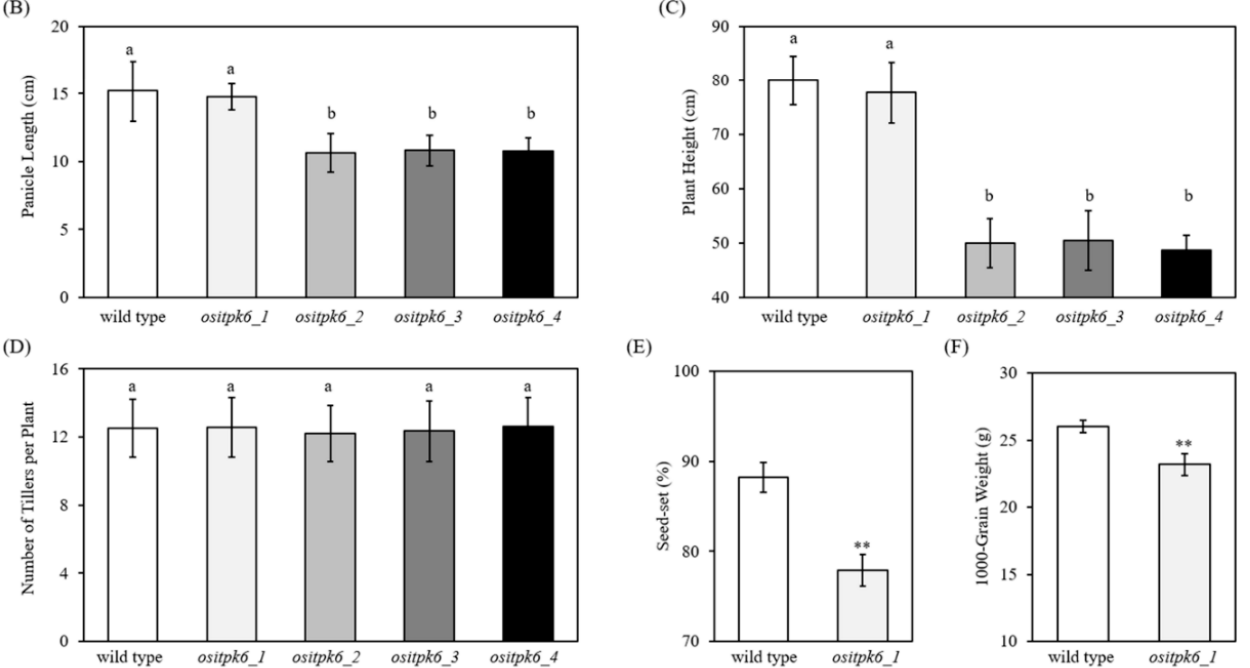

(E)

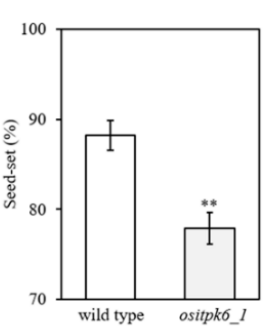

(F)

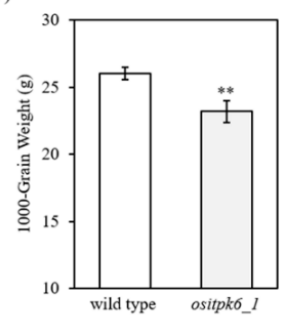

Figure 2. Agronomic traits of mutant OsITPK6 line and wild-type plant. (A) Panicle phenotype of mutant OsITPK6 and wild-type plants. (B-D) Twenty replicates were performed for four OsITPK6 mutant lines and the wild-type plant. Error bars represent the standard error. The different letters show significant differences at a probability of $p<0.05$. (E,F) Twenty replicates were performed for four OsITPK6 mutant lines and the wild type. Error bars represent the standard error. Data with an asterisk(s) are significantly different from those of the wild type $\left({ }^{*} p<0.05,{ }^{* *} p<0.01\right)$. 
(A)

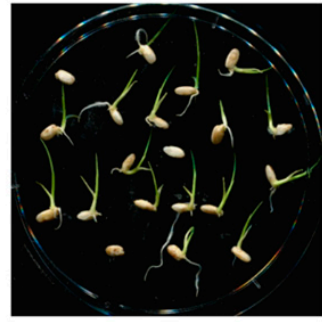

wild type

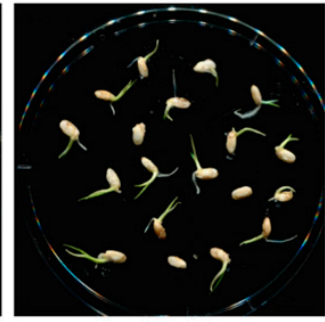

ositpk6_1

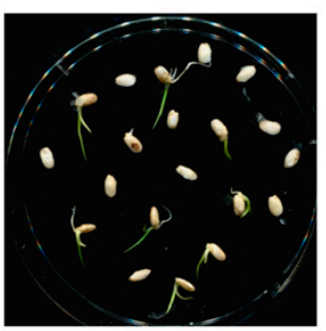

ositpk6_2

(B)

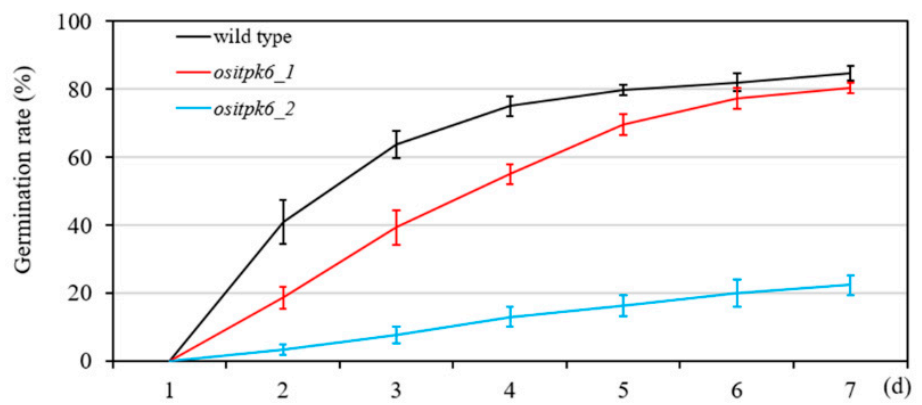

Figure 3. Germination rate of mutant seeds. (A) The pictures were taken on the 5th day after soaking. (B) The germination rate was recorded from 1 to 7 days after soaking, and three replicates were examined in each group.

2.3. Effect of ositpk6 Mutations on Inorganic Phosphorus (Pi), Phytic Acid Phosphorus (PA-P), and Total Phosphorus (TP) in Brown Rice

A colorimetric assay showed that ositpk6_1 and ositpk6_2 had significantly higher Pi levels than the control (Figure 4A). To quantify the mutational effect of ositpk6_1 and ositpk6_2, the Pi, PA-P, and TP contents were assessed in seeds of these two mutant lines, together with Xidao 1 as the WT control. All mutant lines had significantly lower levels of PA-P and higher levels of Pi compared with the control, while TP was not significantly different from that of the control (Figure 4B-D). ositpk6_1 and ositpk6_2 had Pi levels of $1.13 \mathrm{mg} \mathrm{g}^{-1}$ and $1.43 \mathrm{mg} \mathrm{g}^{-1}$, respectively, which were 4.12- and 5.18-fold higher than those of the control $\left(0.28 \mathrm{mg} \mathrm{g}^{-1}\right)$, respectively (Figure 4B). Xidao 1 seeds had a PA-P content of $2.30 \mathrm{mg} \mathrm{g}^{-1}$, which was significantly greater than those of the two mutant lines; the reduction of PA-P levels was $10.1 \%$ and $32.1 \%$ in ositpk6_1 and ositpk6_2, respectively (Figure 4C). Xidao 1 seeds had a TP content of $3.9 \mathrm{mg} \mathrm{g}^{-1}$, which was not significantly different from those of the two mutant lines (Figure 4D).

(A)

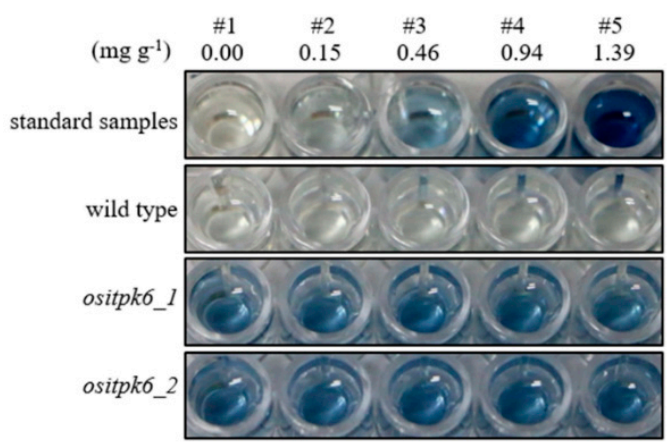

(B)

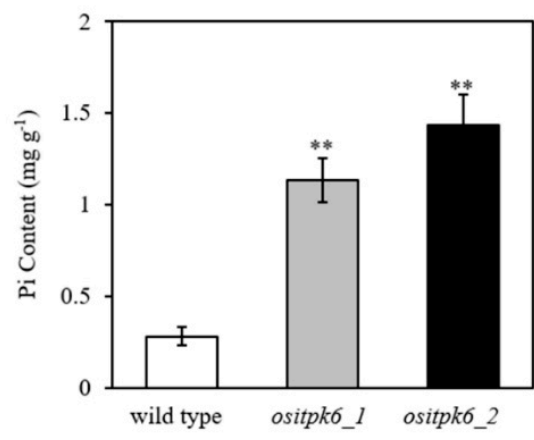

Figure 4. Cont. 
(C)

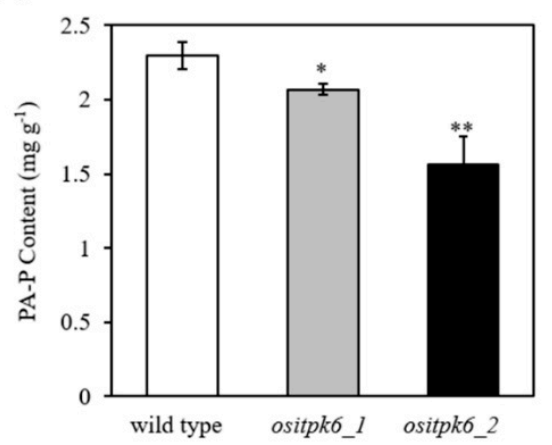

(D)

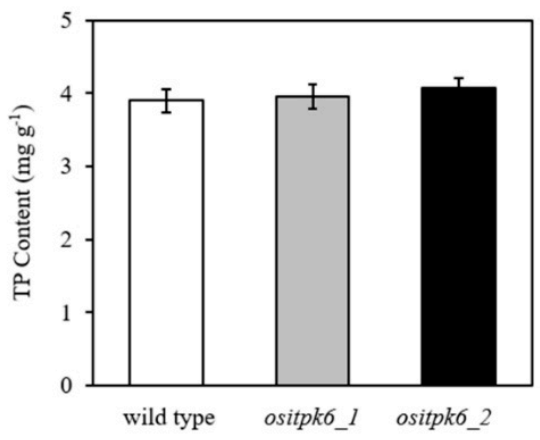

Figure 4. Inorganic $\mathrm{P}(\mathrm{Pi})$, phytic acid $\mathrm{P}(\mathrm{PA}-\mathrm{P})$, and total phosphorus $(\mathrm{TP})$ contents of the mutant OsITPK6 lines and the wild type. (A) Qualitative assay of inorganic phosphorus (Pi) in mutant seeds. The concentration of the Pi standard samples is shown above. Five replicates were performed for two OsITPK6 mutant lines and the wild type. (B-D) Six replicates were performed for two OsITPK6 mutant lines and the wild type. Error bars represent the standard error. Data with an asterisk(s) are significantly different with respect to the wild-type data $\left.{ }^{*} p<0.05,{ }^{* *} p<0.01\right)$.

\subsection{Effect of ositpk6 Mutation on Stress Tolerance}

To further test whether the mutation also had any impact on stress tolerance, we subjected the ositpk6_1 and Xidao 1 plants to osmotic stress treatment (because of the limited number of seeds harvested and the low germination rate, ositpk6_2, was not further analyzed). The growth of ositpk6_1 seedlings appeared to be inferior to that of the WT control grown either under normal or stressed conditions (Figure 5A). The shoot length of ositpk6_1 was shorter than that of the WT with or without stress treatment $(100 \mathrm{mM} \mathrm{NaCl}$ or $20 \mathrm{mM}$ mannitol), while the root length of ositpk6_1 was shorter than that of the WT only under stress (Figure 5A,B). There was no significant difference in the number of leaves and roots with or without stress treatment between ositpk6_1 and WT (Figure 5C).

(A)

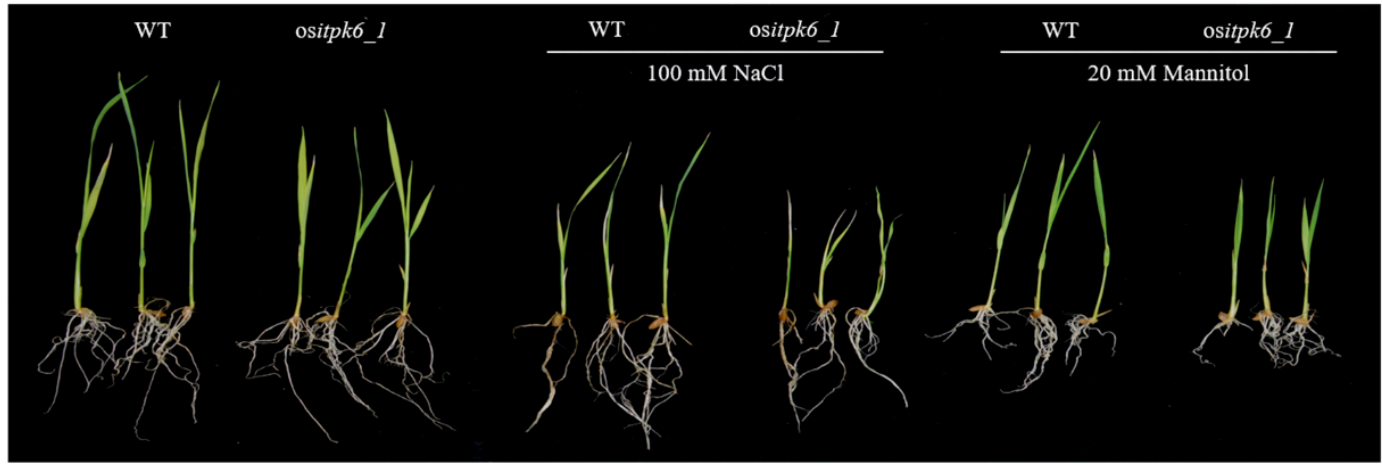

(B)

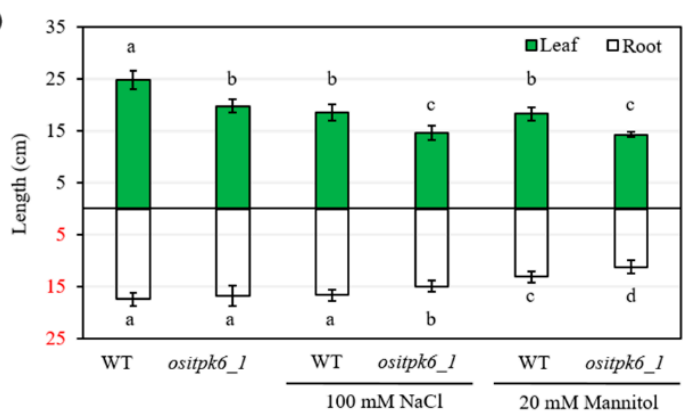

(C)

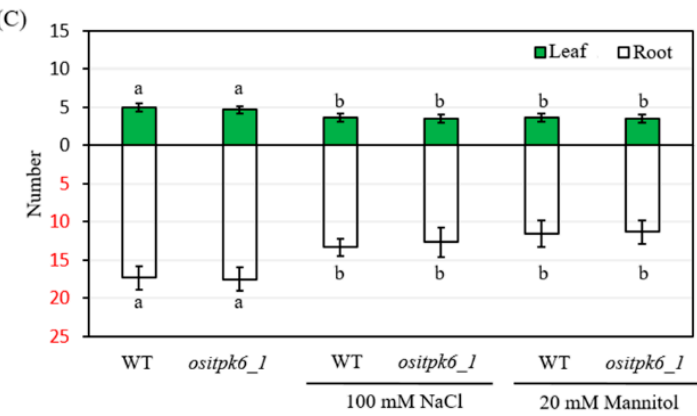

Figure 5. The phenotypes of the mutant OsITPK6_1 and wild type under salt stress $(100 \mathrm{mM} \mathrm{NaCl})$ and drought stress (20 mM mannitol). (A) The picture was taken on the 7th day after treatment. (B,C) Six replicates were performed for the OsITPK6 mutant line and the wild type. Error bars represent the standard error. The different letters show significant differences at a probability of $p<0.05$. 


\section{Discussion}

ITPK6 is a unique gene in the ITPK gene family, and knowledge of its function has so far been very limited. The identification and characterization in rice of two itpk6 mutant lines were reported in a study, which is the only study on the function of ITPK6 in all organisms [30]. Our present study demonstrated that the knockout of OSITPK6 could severely impair plant growth and reproduction, implying that ITPK6 may play important roles in plant growth and development, in addition to the biosynthesis of inositol polyphosphates.

First, we observed that the OsIPTK6 knockout mutants (ositpk6_2,_3, and _4) generated in the present study grew poorly, e.g., their plant height was reduced to almost half of that of the WT, and their fertility was almost abolished (Figure 2). These results suggested that ITPK6 plays an important role not only in the vegetative growth but also in the reproduction of rice. Because the reduction of phytic acid content in ositpk6_1 grains $(-32.1 \%)$ was less than in the P522L mutant line $(-46 \%)$ [30], the reduction of phytic acid alone could not explain the inferior performance of ositpk6_1. Further studies are needed to uncover the biological basis leading to the discrepancy between our present study and that reported in reference [30] regarding the mutational effect on rice growth and reproduction.

In the present study, we identified an ositpk6 mutant, i.e., ositpk6_1, with a 6-bp deletion. Though only two amino acids are expected to be removed from the derived protein, ositpk6_1 did reduce $\mathrm{IP}_{6}$ content. This may be because these two amino acids are located in a conserved region (Figure S1). We previously also observed a similar case of lpa rice, where a 6-bp deletion (and, hence, a deletion of two amino acids) in OsSultr3;3 significantly reduced grain phytic acid content [14]. Although this mutation only reduced phytic acid content by less than $20 \%$, it did exert a negative effect on seed set, grain weight, seed germination, and tolerance to abiotic stresses. This was somehow unexpected, because the P522L mutant line with a $46 \%$ reduction of phytic acid content still had normal plant growth as its WT parent [30]. Further studies are needed to fully evaluate the mutational effect by examining more ositpk6 mutants.

The usefulness of CRISPR/Cas9-based mutagenesis for improving a particular trait is strictly dependent on the performance of the generated mutants. There are often trade-offs for mutating a gene for a specific purpose, and the overall performance of the mutated plant could be affected as a consequence of pleiotropic effects. Enlightened by the findings of Kim and Tai [30], we hoped to establish a fast and effective method for breeding yield-competitive, lpa rice cultivars by using genome-editing techniques. However, our results suggest that OsITPK6 or its product plays an important role in multiple cellular processes, and simply knocking out OsIPTK6 would impair rice growth and reproduction and, hence, would not work for our purpose.

Because [30] of the success in the production of lpa mutants without a significant negative impact on plant growth and seed development, it is still possible to generate ositpk6 mutants without a significant effect on plant growth, if more appropriate vectors can be designed and more mutants are identified and assessed.

In summary, the present study demonstrates that the OSITPK6 gene is essential for rice growth and reproduction.

\section{Materials and Methods}

\subsection{CRISPR/Cas9 Vector Construction and Rice Transformation}

To generate OsITPK6 mutants, the 1st exon of OsITPK6 (Os09g0518700) was chosen as a target (Figure 1A). The sgRNAs were designed by searching UniProt for precise positions (http://www.uniprot. org/), and CRISPR-P program (http://cbi.hzau.edu.cn/cgi-bin/CRISPR/) was used to minimize off-target effects [37]. Because of the homology of the target sequence among OsITPK genes, it is unlikely to cause mutations in the other five OSITPK homolog genes (Figure S2). DNA oligonucleotides were synthesized (Tsingke, Hangzhou, China) for the construction of a CRISPR/Cas9 vector, pH_itpk6, using the pHun4c12s as the starting plasmid, which harbors a CYP81A6-hpRNAi element [38] and 
was modified from pHun4c12 [39]. Correspondingly, the pH_itpk6 plasmid was transformed into Agrobacterium tumefaciens and used for rice transformation.

Rice calli were induced from mature seeds of the cultivar 'Xidao 1' (O. sativa L. japonica) and were transformed with the $\mathrm{pH}$-itpk6 vector by Agrobacterium-mediated transformation according to reference [40]. Transgenic plantlets were regenerated from hygromycin-resistant calli and acclimatized inside a moist growth chamber $\left(28^{\circ} \mathrm{C}\right.$ with a $12 \mathrm{~h}$ photoperiod) for one week before being transplanted to experimental facilities.

Ethics Approval and Consent to Participate: The experiments did not involve endangered or protected species. No specific permits were required for these locations/activities.

\subsection{Mutation Detection in $T_{0}$ Plants}

For detection of transgenes and mutations in regenerated $T_{0}$ plants, total genomic DNA was extracted from leaf tissues following a modified cetyltrimethylammonium bromide (CTAB) method [41]. The presence of the HPT gene was assessed by PCR using the primers HygR-F (5'-AGAAGAAGATGTTGGCGACCT-3') and HygR-R (5'-GTCCTGCGGGTAAATAGCT-3') [42]. Site-specific mutations were detected by $\mathrm{PCR}$ amplification using primer pairs flanking the designated target sites in OsITPK6, i.e., P6-F (5'-CTCGACCCATCCGGTGTTAC-3') and P6-R (5'-AAATCGCAGGGGAGAGATCG-3') (Figure 1A). The following generalized PCR program was used: 5 min at $94^{\circ} \mathrm{C}$, followed by 35 cycles of $30 \mathrm{~s}$ at $94^{\circ} \mathrm{C}, 1 \mathrm{~min}$ at $60^{\circ} \mathrm{C}$, and $1 \mathrm{~min}$ at $72{ }^{\circ} \mathrm{C}$, with a final extension of $10 \mathrm{~min}$ at $72{ }^{\circ} \mathrm{C}$.

The PCR products were first subjected to HRM analysis for mutations according to reference [43], putative mutants were sequenced (TSINGKE, Hangzhou, China), and mutated sequences were decoded using the DSDecode program (http://skl.scau.edu.cn/dsdecode/) [44]. Mutations of a few selected plants were further confirmed by clone sequencing.

\subsection{Development of Transgene-Free Mutant Lines}

To obtain homozygous transgene-free mutants, $\mathrm{T}_{1}$ seedlings were foliar-sprayed with $1000 \mathrm{mg} / \mathrm{L}$ bentazon (approximately $100 \mathrm{~mL} / \mathrm{m}^{2}$ ) at about the four-leaf stage according to a previous study [38]. At least five surviving $T_{1}$ plants from each independent $T_{0}$ plants were selected for further analysis of the presence of T-DNA and site-specific mutations. Eventually, four OsITPK6 mutant lines (ositpk6_1, _2, 3 , and _4) (Figure 1A) were identified; the $T_{2}$ and advanced-generation seeds were used for the experiments.

\subsection{Agronomic Traits Assay}

The transgene-free mutant lines and their wild-type parent control (Xidao 1) were grown at the Experimental Farm of Zhejiang Zhijiang Seed Tec. Ltd., Hangzhou, China, during the summer season, and their agronomic traits were evaluated either in fields or post-harvest. Plants were grown in randomized plots, each with 60 plants. Twenty inner plants of each plot were evaluated for each parameter.

\subsection{Seed Germination Assay}

A controlled germination test was performed to assess seed germination capability, each with 100 seeds and replicated for three times [45]. Seeds were soaked in water for $48 \mathrm{~h}$ at $30^{\circ} \mathrm{C}$ and then germinated on filter paper soaked with distilled water at $30^{\circ} \mathrm{C}$ in the dark for one week, and the germination percentage was recorded daily.

\subsection{Seed Phosphorus Assay}

Seed inorganic $\mathrm{P}(\mathrm{Pi})$ levels were assayed qualitatively according to the micro-determination method developed in reference [46] with modifications. The qualitative assay was used for the 
identification of the high-inorganic-P (HIP) phenotype. The seeds were transferred to 96-well plates and extracted in $0.4 \mathrm{M} \mathrm{HCl}$ solution $\left(10 \mu \mathrm{L}\right.$ per mg sample) overnight at $4{ }^{\circ} \mathrm{C}$. Aliquots of $10 \mu \mathrm{L}$ of supernatant per each sample were used for Pi level determination, according to reference [4] with slight modifications [6]. The development of a blue color implied increased level of Pi (HIP), while colorless samples typified the WT levels of parent varieties (Figure S1).

Seed Pi levels were also quantitatively determined according to reference [47] in triplicates, as follows. Brown rice grains were ground into rice flour, and $\sim 400 \mathrm{mg}$ rice flour per sample were extracted in $12.5 \%(w / v)$ TCA (trichloroacetic acid containing $25 \mathrm{mM} \mathrm{MgCl}_{2}$ ) by gentle shaking overnight at $4{ }^{\circ} \mathrm{C}$. After centrifugation at $15,000 \times g$ for $10 \mathrm{~min}$, the supernatants were used for Pi assay according to reference [2].

The PA content was determined for brown rice flour according to reference [48] by using a commercial assay kit (Megazyme, Irland) in triplicate. Briefly, $\sim \mathrm{g}$ of brown rice grains was mixed with $20 \mathrm{~mL}$ of hydrochloric acid $(0.66 \mathrm{M})$, stirred vigorously for $3 \mathrm{~h}$ at room temperature, and then centrifuged at 13,000 rpm for $10 \mathrm{~min}$. Then, $0.5 \mathrm{~mL}$ of the supernatant was immediately neutralized by the addition of $0.5 \mathrm{~mL}$ of sodium hydroxide solution $(0.75 \mathrm{M})$. The neutralized sample extract was subjected to an enzymatic dephosphorylation reaction procedure. The absorbances at $655 \mathrm{~nm}$ was determined using an ultraviolet spectrophotometer.

Total seed phosphorus and mineral elements were determined according to reference [49] in triplicate. Briefly, brown rice samples were digested in a microwave digestion system (Mars6, USA) at $160^{\circ} \mathrm{C}$ for $40 \mathrm{~min}$, using $200 \mathrm{mg}$ of sample in $7 \mathrm{~mL}$ of $\mathrm{HNO}_{3}$. The digested solution was concentrated at $140{ }^{\circ} \mathrm{C}$ for $2 \mathrm{~h}$ until less than $1 \mathrm{~mL}$ of solution was left and then brought to $30 \mathrm{~mL}$ with ultrapure water [50]. Total seed phosphorus and mineral elements were analyzed by Inductively Coupled Plasma-Mass Spectrometry (ICP-MS) (PerkinElmer, MA, USA).

\subsection{Stress Treatment}

For stress treatment, 14-day-old seedlings were planted in 1× Murashige and Skoog (MS) liquid medium [51], supplemented with $100 \mathrm{mM} \mathrm{NaCl}$ or $20 \mathrm{mM}$ mannitol and grown for 7 days before sampling at midday.

\subsection{Statistical Analysis}

All statistical analyses were performed using the student's $t$-test. The experimental data are presented with the mean standard errors (SE) based on three to six replications. The means were compared by ANOVA, and the significance of the differences between group means were calculated by the Bonferroni Post-tests.

Supplementary Materials: The following are available online at http://www.mdpi.com/2223-7747/8/5/114/s1. Figure S1: Multiple sequence alignment of ITPK6s. All the data are from RAP-DB (http://rapdb.dna.affrc.go.jp/) and Gramene (http://www.gramene.org/). Analysis of ITPK6 sequences from six organisms using BioEdit software. Identical amino acid residues are boxed in same color. Short lines indicate gaps introduced during alignment. The mutant site of OsITPK6 is marked by a redbox. Figure S2. Multiple sequence alignment of OsITPKs. All data are from RAP-DB (http://rapdb.dna.affrc.go.jp/) and Gramene (http://www.gramene.org/). Analysis of OsITPK1, OsITPK2, OsITPK3, OsITPK4, OsITPK5, and OsITPK6 sequences using the BioEdit software. Identical DNA bases are in the same color. Dotted lines indicate gaps introduced during alignment. The target site of OsITPK6 is marked by a red box.

Author Contributions: Q.S. and M.J. planned and designed the research. M.J., Y.L. (Yang Liu), Y.L. (Yanhua Liu), and Y.T. performed the laboratory experiments. M.J., Y.L. (Yang Liu), and Q.S. analyzed the data together. M.J. finished the first draft, which J.H. and Q.S. edited and converted into the final draft. All authors reviewed and approved the final manuscript.

Funding: This work was supported by the Zhejiang Provincial S\&T Project on Breeding of Agricultural (Food) Crops (Grant No. 2016C02050-2).

Acknowledgments: We thank workers in the farms of Zhejiang Zhijiang Seed Tec. Ltd. for taking care of the paddy fields. We also appreciate the assistance in total seed phosphorus and mineral elements measurement of Wu Zhongchang and Xu Jiming from the College of Life Sciences, Zhejiang University, Hangzhou, China. 
Conflicts of Interest: The authors declare no conflict of interest.

\section{References}

1. Lott, J.N.A.; Ockenden, I.; Raboy, V.; Batten, G.D. Phytic acid and phosphorus in crops seeds and fruits: A global estimate. Seed Sci. Res. 2000, 10, 11-33.

2. Raboy, V.; Young, K.A.; Dorsch, J.A.; Cook, A. Genetics and breeding of seed phosphorus and phytic acid. J. Plant Physiol. 2001, 158, 489-497. [CrossRef]

3. Raboy, V.; Gerbasi, P.F.; Young, K.A.; Stoneberg, S.D.; Pickett, S.G.; Bauman, A.T.; Murthy, P.P.N.; Sheridan, W.F.; Ertlet, D.S. Origin and seed phenotype of maize low phytic acid 1-1 and low phytic acid 2-1. Plant Physiol. 2000, 124, 355-368. [CrossRef] [PubMed]

4. Larson, S.R.; Rutger, J.N.; Young, K.A.; Raboy, V. Isolation and genetic mapping of a non-lethal rice (Oryza sativa L.) low phytic acid 1 mutation. Crop Sci. 2000, 40, 1397-1405. [CrossRef]

5. Kim, S.I.; Andaya, C.B.; Newman, J.W.; Goyal, S.S.; Tai, T.H. Isolation and characterization of a low phytic acid rice mutant reveals a mutation in the rice orthologue of maize MIK. Theor. Appl. Genet. 2008, 117, 1291-1301. [CrossRef] [PubMed]

6. Liu, Q.L.; Xu, X.H.; Ren, X.L.; Fu, H.W.; Wu, D.X.; Shu, Q.Y. Generation and characterization of low phytic acid germplasm in rice (Oryza sativa L.). Theor. Appl. Genet. 2007, 114, 803-814. [CrossRef]

7. Zhao, H.J.; Liu, Q.L.; Fu, H.W.; Xu, X.H.; Wu, D.X.; Shu, Q.Y. Effect of non-lethal low phytic acid mutations on grain yield and seed viability in rice. Field Crop. Res. 2008, 108, 206-211. [CrossRef]

8. Raboy, V. Approaches and challenges to engineering seed phytate and total phosphorus. Plant Sci. 2009, 177, 281-296. [CrossRef]

9. Xu, X.H.; Zhao, H.J.; Liu, Q.L.; Frank, T.; Engel, K.H.; An, G.; Shu, Q.Y. Mutations of the multi-drug resistance-associated protein $\mathrm{ABC}$ transporter gene 5 result in reduction of phytic acid in rice seeds. Theor. Appl. Genet. 2009, 119, 75-83. [CrossRef]

10. Ali, N.; Paul, S.; Gayen, D.; Sarkar, S.N.; Datta, K.; Datta, S.K. Development of low phytate rice by RNAi mediated seed specific seed specific silencing of inositol 1,3,4,5,6-pentakisphosphate 2-kinase gene (IPK1). PLoS ONE 2013, 8, e68161. [CrossRef]

11. Zhao, H.J.; Cui, H.R.; Xu, X.H.; Tan, Y.Y.; Fu, J.J.; Liu, G.Z.; Poirier, Y.; Shu, Q.Y. Characterization of OsMIK in a rice mutant with reduced phytate content reveals an insertion of a rearranged retrotransposon. Theor. Appl. Genet. 2013, 126, 3009-3020. [CrossRef]

12. Li, W.X.; Huang, J.Z.; Zhao, H.J.; Tan, Y.Y.; Cui, H.R.; Poirier, Y.; Shu, Q.Y. Production of low phytic acid rice by hairpin RNA- and artificial microRNA-mediated silencing of OsMIK in seeds. Plant Cell Tiss. Org. 2014, 119, 15-25. [CrossRef]

13. Li, W.X.; Zhao, H.J.; Pang, W.Q.; Cui, H.R.; Poirier, Y.; Shu, Q.Y. Seed-specific silencing of OsMRP5 reduces seed phytic acid and weight in rice. Transgenic Res. 2014, 23, 585-599. [CrossRef] [PubMed]

14. Zhao, H.J.; Frank, T.; Tan, Y.Y.; Zhou, C.G.; Jabnoune, M.; Arpat, A.B.; Cui, H.R.; Huang, J.Z.; He, Z.H.; Poirier, Y.; et al. Disruption of OsSULTR3;3 reduces phytate and phosphorus concentrations and alters the metabolite profile in rice grains. New Phytol. 2016, 211, 926-939. [CrossRef] [PubMed]

15. Guttieri, M.; Bowen, D.; Dorsch, J.A.; Raboy, V.; Souza, E. Identification and characterization of a low phytic acid wheat. Crop Sci. 2014, 44, 418-424. [CrossRef]

16. Pilu, R.; Panzeri, D.; Gavazzi, G.; Rasmussen, S.K.; Consonni, G.; Nielsen, E. Phenotypic, genetic and molecular characterization of a maize low phytic acid mutant (lpa 241). Theor. Appl. Genet. 2003, 107, $980-987$. [CrossRef] [PubMed]

17. Shi, J.; Wang, H.; Hazebroek, J.; Ertl, D.S.; Harp, T. The maize low-phytic acid 3 encodes a myo-inositol kinase that plays a role in phytic acid biosynthesis in developing seeds. Plant J. 2005, 42, 408-419. [CrossRef] [PubMed]

18. Suzuki, M.; Tanaka, K.; Kuwano, M.; Yoshida, K.T. Expression pattern of inositol phosphate-related enzymes in rice (Oryza sativa L.): Implications for the phytic acid biosynthetic pathway. Gene 2007, 405, 55-64. [CrossRef] [PubMed]

19. Takazawa, K.; Perret, J.; Dumont, J.E.; Erneux, C. Molecular cloning and expression of a new putative inositol 1, 4, 5-trisphosphate 3-kinase isoenzyme. Biochem. J. 1991, 278, 883-886. [CrossRef] 
20. Wilson, M.P.; Majerus, P.W. Characterization of a cDNA encoding Arabidopsis thaliana inositol 1, 3, 4-trisphosphate 5/6-kinase. Biochem. Biophys. Res. Commun. 1997, 232, 678-681. [CrossRef]

21. Sweetman, D.; Stavridou, I.; Johnson, S.; Green, P.; Caddick, S.E.; Brearley, C.A. Arabidopsis thaliana inositol 1, 3, 4-trisphosphate 5/6-kinase 4 (AtITPK4) is an outlier to a family of ATP-grasp fold proteins from Arabidopsis. FEBS Lett. 2007, 581, 4165-4171. [CrossRef]

22. Field, J.; Wilson, M.P.; Mai, Z.; Majerus, P.W.; Samuelson, J. An Entamoeba histolytica inositol 1, 3, 4-trisphosphate 5/6-kinase has a novel 3-kinase activity. Mol. Biochem. Parasitol. 2000, 108, 119-123. [CrossRef]

23. Qin, Z.X.; Chen, Q.J.; Tong, Z.; Wang, X.C. The Arabidopsis inositol 1, 3, 4-trisphosphate 5/6 kinase, AtItpk-1, is involved in plant photomorphogenesis under red light conditions, possibly via interaction with COP9 signalosome. Plant Physiol. Biochem. 2005, 43, 947-954. [CrossRef]

24. Kuo, H.F.; Hsu, Y.Y.; Lin, W.C.; Chen, K.Y.; Munnik, T.; Brearley, C.A.; Chiou, T.J. Arabidopsis inositol phosphate kinases ipk1 and itpk1 constitute a metabolic pathway in maintaining phosphate homeostasis. Plant J. 2018, 95. [CrossRef] [PubMed]

25. Tang, Y.; Tan, S.; Xue, H. Arabidopsis inositol 1,3,4-trisphosphate 5/6 kinase 2 is required for seed coat development. ActaBiochim. Biophys. Sin. 2013, 45, 549-560. [CrossRef]

26. Krishnan, V.; Jain, P.; Vinutha, T.; Hada, A.; Manickavasagam, M.; Ganapathi, A.; Raj, D.R.; Archana, S. Molecular modeling and 'in-silico' characterization of 'Glycine max' inositol $(1,3,4)$ tris 5/6 kinase-1(Gmitpk1) - A potential candidate gene for developing low phytate transgenics. Plant Omics. 2015, 8, 381-391.

27. Marathe, A.; Krishnan, V.; Vinutha, T.; Dahuja, A.; Jolly, M.; Sachdev, A. Exploring the role of inositol 1, 3, 4trisphosphate 5/6 kinase-2 (Gmitpk2) as a dehydration and salinity stress regulator in Glycine max (L.) merr through heterologous expression in E.coli. Plant Physiol. Biochem. 2017, 8, 50. [CrossRef] [PubMed]

28. Niu, X.; Chen, Q.; Wang, X. Ositl1 gene encoding an inositol 1,3,4-trisphosphate 5/6-kinase is a negative regulator of osmotic stress signaling. Biotechnol. Lett. 2008, 30, 1687-1692. [CrossRef] [PubMed]

29. Du, H.; Liu, L.H.; You, L.; Yang, M.; He, Y.B.; Li, X.H.; Xiong, L.Z. Characterization of an inositol 1,3,4-trisphosphate 5/6-kinase gene that is essential for drought and salt stress responses in rice. Plant Mol. Biol. 2011, 77, 547-563. [CrossRef]

30. Kim, S.I.; Tai, T.H. Identification of novel rice low phytic acid mutations via TILLING by sequencing. Mol. Breed. 2014, 34, 1717-1729. [CrossRef]

31. Li, J.; Zhang, Y.; Chen, K.; Liang, Z. Targeted genome modification of crop plants using a CRISPR/CAS system. Nat. Biotechnol. 2013, 31, 686-688.

32. Cao, H.X.; Wang, W.; Le, H.T.T.; Vu, G.T.H. The power of CRISPR/Cas9-induced genome editing to speed up plant breeding. Int. J. Genom. 2016, 10, 5078796. [CrossRef] [PubMed]

33. Kumlehn, J.; Pietralla, J.; Hensel, G.; Pacher, M.; Puchta, H. The CRISPR/Cas revolution continues: From efficient gene editing for crop breeding to plant synthetic biology. J. Integr. Plant Biol. 2018, 60, 12. [CrossRef]

34. Jung, C.; Capistrano-Gossmann, G.; Braatz, J.; Sashidhar, N.; Melzer, S. Recent developments in genome editing and applications in plant breeding. Plant Breed. 2017, 137. [CrossRef]

35. Lu, H.P.; Luo, T.; Fu, H.W.; Wang, L.; Tan, Y.Y.; Huang, J.Z.; Wang, Q.; Ye, G.Y.; Gatehouse, A.M.R.; Lou, Y.G.; et al. Resistance of rice to insect pests mediated by suppression of serotonin biosynthesis. Nat. Plants 2018, 4, 338-344. [CrossRef]

36. Liu, S.M.; Jiang, J.; Liu, Y.; Meng, J.; Xu, S.L.; Tan, Y.Y.; Li, Y.F.; Shu, Q.Y.; Huang, J.Z. Characterization and Evaluation of OsLCT1 and OsNramp5 Mutants Generated Through CRISPR/Cas9-Mediated Mutagenesis for Breeding Low Cd Rice. Rice Sci. 2019, 26, 88-97.

37. Lei, Y.; Lu, L.; Liu, H.Y.; Li, S.; Xing, F.; Chen, L.L. CRISPR-P: A web tool for synthetic single-guide RNA design of CRISPR-system in plants. Mol. Plant 2014, 7, 1494-1496. [CrossRef] [PubMed]

38. Lu, H.P.; Liu, S.M.; Xu, S.L.; Chen, W.Y.; Zhou, X.; Tan, Y.Y.; Huang, J.Z.; Shu, Q.Y. CRISPR-S: An active interference element for a rapid and inexpensive selection of genome-edited, transgene-free rice plants. Plant Biotechnol. J. 2017, 15, 1371-1373. [CrossRef]

39. Xu, R.F.; Li, H.; Qin, R.Y.; Wang, L.; Li, L.; Wei, P.C.; Yang, J.B. Gene targeting using the Agrobacterium tumefaciens-mediated CRISPR-Cas system in rice. Rice 2014, 7, 5. [CrossRef] [PubMed] 
40. Li, W.X.; Wu, S.L.; Liu, Y.H.; Jin, G.L.; Zhao, H.J.; Fan, L.J.; Shu, Q.Y. Genome-wide profiling of genetic variation in Agrobacterium-transformed rice plants. J. Zhejiang Univ. Sci. B 2016, 17, 992-996. [CrossRef] [PubMed]

41. Zhang, H.L.; Huang, J.Z.; Chen, X.Y.; Tan, Y.Y.; Shu, Q.Y. Competitive amplification of differentially melting amplicons facilitates efficient genotyping of photoperiod-and temperature-sensitive genic male sterility in rice. Mol. Breed. 2014, 34, 1765-1776. [CrossRef]

42. Li, W.L.; Xu, B.B.; Song, Q.J.; Liu, X.M.; Xu, J.M.; Brookes, P.C. The identification of 'hotspots' of heavy metal pollution in soil-rice systems at a regional scale in eastern China. Sci. Total Environ. 2014, 472, 407-420. [CrossRef]

43. Li, S.; Liu, S.M.; Liu, Y.H.; Lu, H.P.; Tan, Y.Y.; Huang, J.Z.; Wei, P.C.; Shu, Q.Y. HRM-facilitated rapid identification and genotyping of mutations induced by CRISPR/Cas9 mutagenesis in rice. Crop Breed. Appl. Biotechnol. 2018, 18, 184-191. [CrossRef]

44. Liu, W.Z.; Xie, X.R.; Ma, X.L.; Li, J.; Chen, J.H.; Liu, Y.G. DSDecode: Aweb-based tool for decoding of sequencing chromatograms for genotyping of targeted mutations. Mol. Plant 2015, 8, 1431-1433. [CrossRef]

45. Campion, B.; Sparvoli, F.; Doria, E.; Tagliabue, G.; Galasso, I.; Fileppi, M.; Bollini, R.; Nielsen, E. Isolation and characterization of an lpa (low phytic acid) mutant in common bean (Phaseolus vulgaris L.). Theor. Appl. Genet. 2009, 118, 1211-1221. [CrossRef]

46. Chen, P.S.; Toribara, T.Y.; Warner, H. Micro determination of phosphorous. Anal. Chem. 1956, 28, $1756-1758$. [CrossRef]

47. Wilcox, J.R.; Premachandra, G.S.; Young, K.A.; Raboy, V. Isolation of high seed inorganic P, low-phytate soybean mutants. Crop Sci. 2000, 40, 1601-1605. [CrossRef]

48. McKie, V.A.; McCleary, B.V.A. Novel and rapid colorimetric method for measuring total phosphorus and phytic acid in foods and animal feeds. J. AOAC Int. 2016, 99, 738-743. [CrossRef]

49. Hu, L.F.; McBride, M.B.; Cheng, H.; Wu, J.J.; Shi, J.C.; Xu, J.M.; Wu, L.S. Root-induced changes to cadmium speciation in the rhizosphere of two rice (Oryza sativa L.) genotypes. Environ. Res. 2011, 111, 356-361. [CrossRef]

50. Meng, J.; Zhong, L.B.; Wang, L.; Liu, X.M.; Tang, C.X.; Chen, H.L.; Xu, J.M. Contrasting effects of alkaline amendments on the bioavailability and uptake of $\mathrm{Cd}$ in rice plants in a Cd-contaminated acid paddy soil. Environ. Sci. Pollut. R. 2018, 25, 8827-8835. [CrossRef]

51. Murashige, T.; Skoog, F. A revised medium for rapid growth and bio assays with tobacco tissue cultures. Physiol. Plantarum 1962, 15, 473-497. [CrossRef] 